\title{
Primary Intrathoracic Goiter
}

\author{
${ }^{1}$ Dorin T Colibaseanu, ${ }^{2}$ Jon A van Heerden, ${ }^{3}$ David R Farley, ${ }^{4}$ Stephen D Cassivi \\ ${ }^{1,4}$ Division of General Thoracic Surgery, Mayo Clinic, Rochester, Minnesota \\ ${ }^{2,3}$ Department of Surgery, Mayo Clinic, Rochester, Minnesota
}

Correspondence: Stephen D Cassivi, Division of General Thoracic Surgery, Mayo Clinic, 200 First Street SW, Rochester Minnesota-55902

\begin{abstract}
The differential diagnosis of a middle mediastinal mass is fairly broad. Though relatively rare, the diagnosis of primary intrathoracic goiter is a clinically important consideration that should not be overlooked. The nature of this clinical entity and its diagnostic implications are discussed. Relevant radiographic imaging is evaluated demonstrating the key findings. Indications for intervening and treatment options are reviewed. Specifically, the presence of a growing substernal goiter in an otherwise fit patient is generally an indication for surgical resection. The nature of a primary intrathoracic goiter usually obviates the more common transcervical approach since by definition the mediastinal goiter is discontinuous with the cervical thyroid gland. Other transthoracic approaches are therefore necessary.
\end{abstract}

Keywords: Goiter, intrathoracic, substernal, mediastinum, thyroid, mass.

\section{CLINICAL SCENARIO}

A 51 year-old-female was incidentally found to have a mediastinal mass on a routine chest radiograph in February, 2006 (Fig. 1). Subsequent computerized chest tomography (CT) demonstrated a $6.3 \times 4.8 \mathrm{~cm}$, irregular and enhancing right suprahilar mass displacing the trachea to the left and extending inferiorly to the carina. A transthoracic needle biopsy was performed showing thyroid follicles with colloid consistent with a mediastinal goiter. As she was asymptomatic, the patient opted for continued surveillance. Of note, the patient had previously undergone a left thyroid lobectomy for a benign goiter in 1987.

In May, 2009 a repeat chest CT scan showed the mediastinal mass to have increased in size to $8.0 \times 6.5 \mathrm{~cm}$. The mass was discontinuous with the residual right lobe of the thyroid (Figs $2 \mathrm{~A}$ and $\mathrm{B})$. Other than occasional dysphagia, the patient remained otherwise asymptomatic.

Surgical resection of the mass was performed via a limited muscle sparing right thoracotomy. The mass was resected intact from the middle mediastinum where it was not invading but closely juxtaposed to the superior vena cava anteriorly, azygous vein inferiorly, and trachea medially (Figs $3 \mathrm{~A}$ and B). The blood supply was from segmental arterial branches of the ascending aorta. Final pathologic evaluation confirmed a benign ectopic thyroid mass weighing 179 grams (Figs 4A and B). The patient was discharged home on the third postoperative day. Her dysphagia has improved and her recovery has been uneventful.

\section{DISCUSSION}

The differential diagnosis for a middle mediastinal mass can be broadly divided in malignant and nonmalignant lesions. Malignant masses include lymphoma, lymphatic metastases,

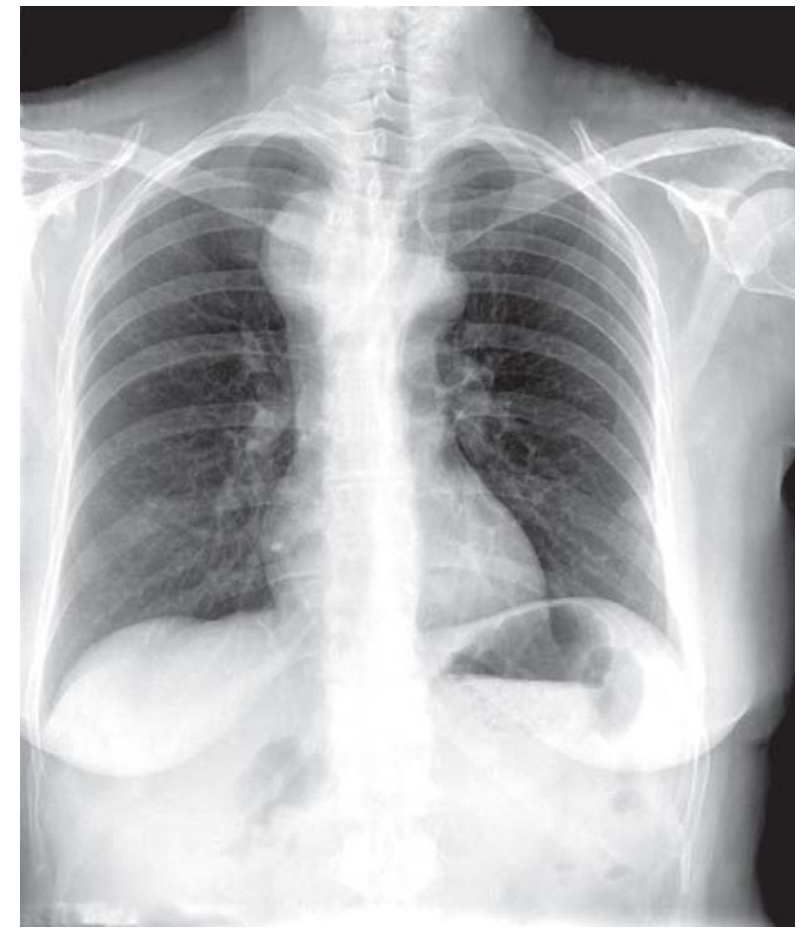

Fig. 1: Initial screening chest $\mathrm{X}$-ray shows a right-sided mediastinal mass

and tracheal tumors. Nonmalignant masses can be further categorized as infectious (lymphadenopathy due to tuberculosis or fungal infections), inflammatory (sarcoidosis, silicosis, pneumoconiosis), vascular (aortic aneurysm, ectatic azygous vein), or congenital (esophageal or bronchial related foregut cysts). Rarely, thyroid masses can be found in the middle 

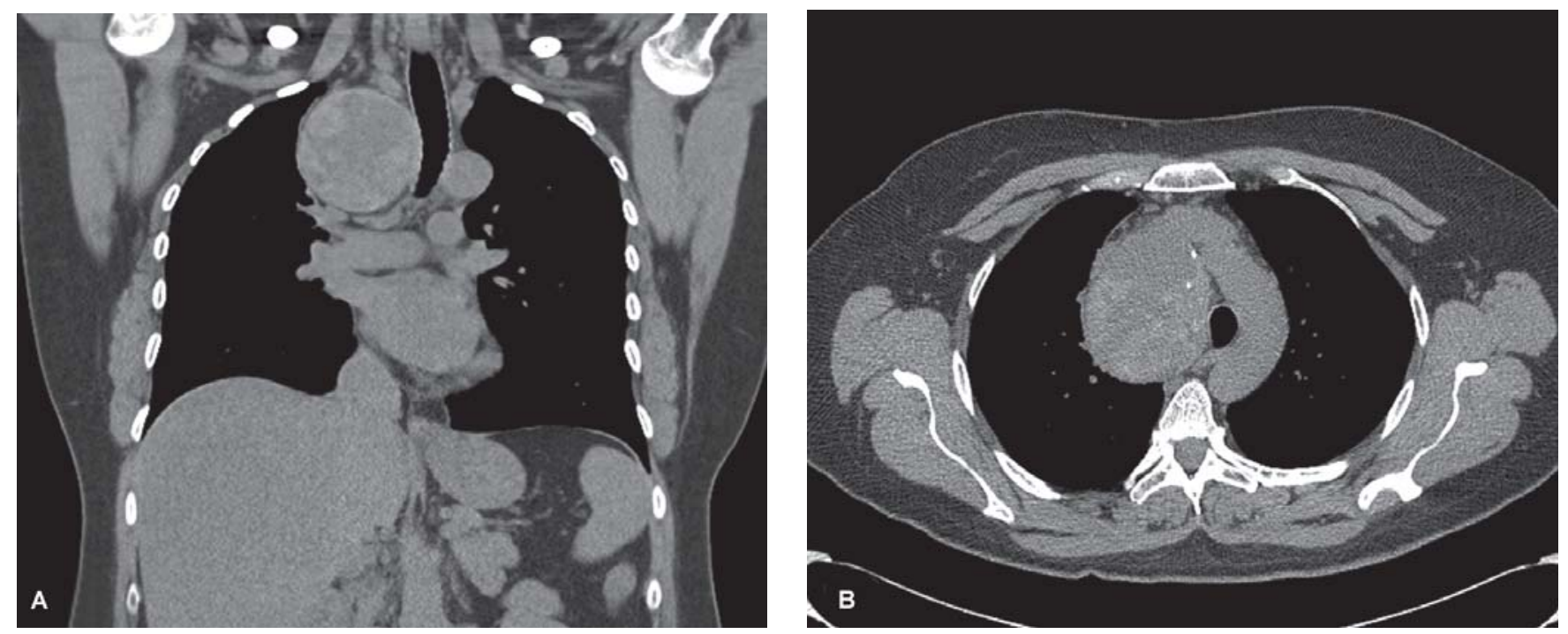

Figs 2A and B: Computed tomography of the chest demonstrating the primary intrathoracic goiter in the middle mediastinum on (A) coronal and (B) axial views
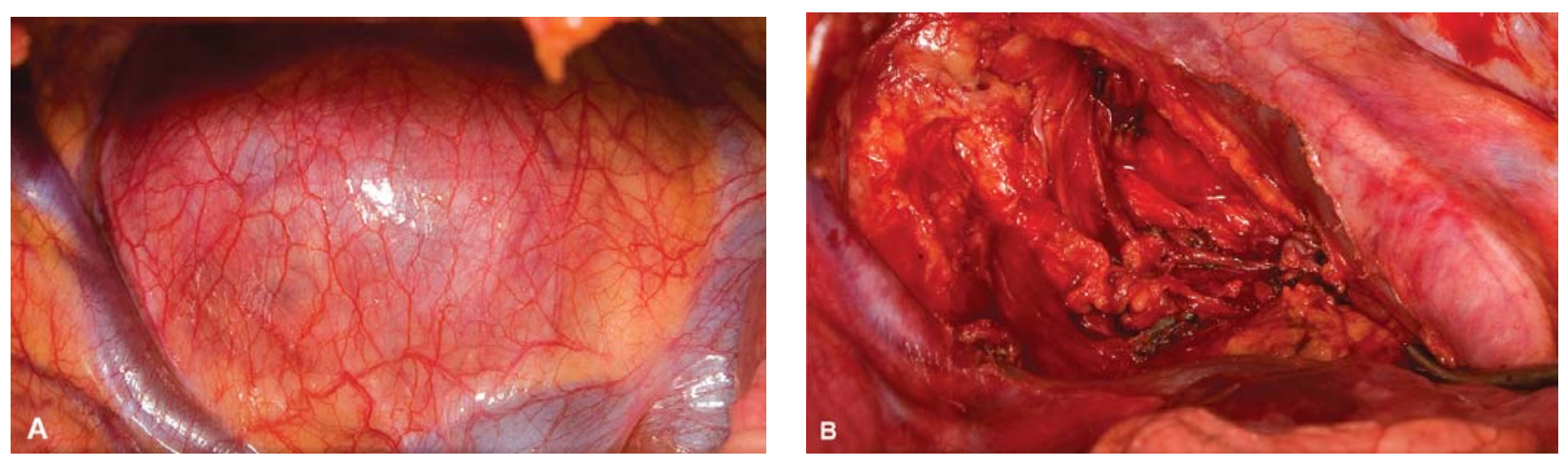

Figs 3A and B: Intraoperative images of the primary intrathoracic goiter showing; (A) the mass beneath the mediastinal pleura abutting the superior vena cava anteriorly and the azygous vein inferiorly prior to dissection; (B) the operative field following resection with clips on the goiter's segmental blood supply from the ascending aorta

mediastinum, though they are more commonly seen in the anterior mediastinum.

The presence of a growing substernal goiter in a patient fit to undergo general anesthesia is an indication for surgery. ${ }^{1}$ Even in asymptomatic patients, these masses will grow, albeit slowly, and will inevitably lead to compression of nearby structures, including the trachea, esophagus, or surrounding vasculature and nerves. Of particular concern is the uncommon but clinically significant possibility of acute airway obstruction due to goiter hemorrhage (traumatic or spontaneous).

This case illustrates a primary intrathoracic goiter in that the intrathoracic thyroid mass was discontinuous with the normal cervical thyroid tissue. ${ }^{2}$ Distinction between primary and secondary goiters is important since a primary goiter in the presence of a normal or goitrous cervical thyroid gland can be independently affected by malignancy. Primary intrathoracic goiters commonly reach greater sizes compared to more common secondary intrathoracic goiters which retain anatomic continuity with the cervical thyroid gland. Due to its location, an intrathoracic goiter is also not accessible for physical examination with the only way to adequately follow these patients being periodic radiographic imaging.

Because of their progressive growth, potential for harboring occult malignancy, and rare but important complications if left untreated, surgical resection is recommended for patients with primary intrathoracic goiters. By definition, the primary intrathoracic goiter is discontinuous from the cervical thyroid gland and therefore is not amenable to a conventional transcervical approach which is the preferred approach for continuous goiters emanating from the neck and extending into the chest. The size and location of the goitrous mass will determine the options for surgical approach which include thoracoscopy, limited thoracotomy or sternotomy. ${ }^{3}$ Once resected, no further treatment is necessary for benign primary intrathoracic goiters other than standard postoperative care. 

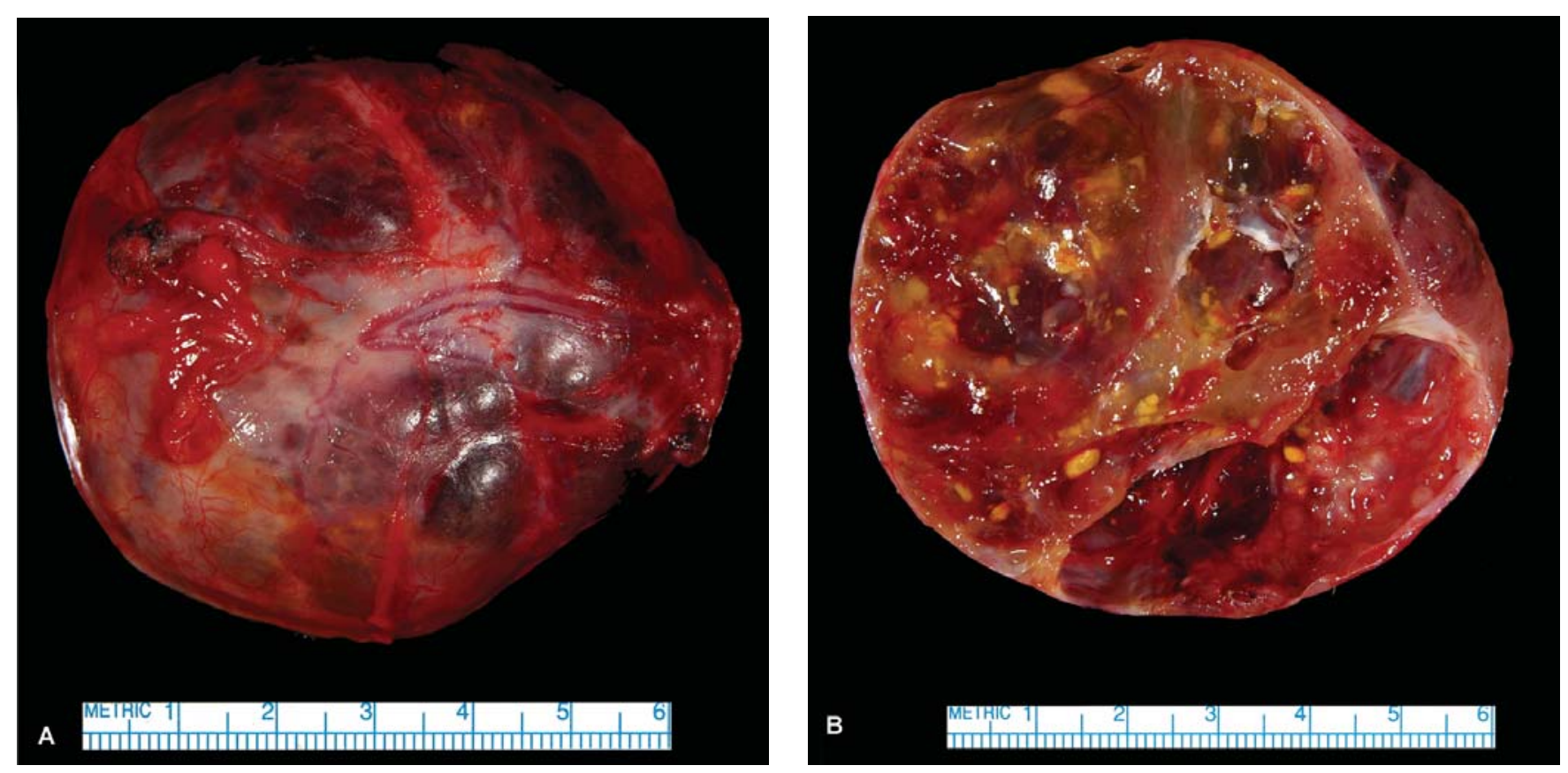

Figs 4A and B: Gross pathology images of the resected primary intrathoracic goiter; (A) Uncut; (B) Transverse section

\section{REFERENCES}

1. de Perrot M, Fadel E, Mercier O, Farhamand P, Fabre D, et al. Surgical management of mediastinal goiters: When is a sternotomy required? Thorac Cardiovasc Surg 2007;55:39-43.
2. Foroulis CN, Rammos KS, Sileli MN, Papakonstantinou C. Primary intrathoracic goiter: A rare and potentially serious entity. Thyroid 2009;19(3):213-18.

3. Van Heerden JA. Teaspoon or tablespoon? Surgery 2003;133:336. 